\title{
Research of Ship Autopilot Rudder Based on Deep Belief Network
}

\author{
Li Shaowei ${ }^{1+}$, Wang Shengzheng ${ }^{2}$ \\ ${ }^{1}$ School of Mathematics and Computer Science, Jianghan University, Hubei, China \\ ${ }^{2}$ Merchant Marine College, Shanghai Maritime University, Shanghai, China
}

\begin{abstract}
In order to improve the control precision of the existing ship autopilot and improve the adaptive capability of the autopilot, an autopilot control algorithm based on the deep confidence network (DBN) is proposed. First of all, using the contrast divergence algorithm and the data recorded in the examination system of the Shanghai Maritime University, the constrained Boltzmann machines (RBMs) that make up each DBN are pre-trained in turn, and the results are used as the depth nerve Network weight of the initial value. On this basis, the back propagation algorithm is used to fine-tune the multi-layer depth structure. The simulation results show that the simulated sailing error between this method and the master captain is only $5.2 \%$.
\end{abstract}

Keywords: Autopilot Rudder, Deep Neural Networks, CD Algorithm, RBM, BP, Training

\section{Introduction}

With the development of artificial intelligence, neural networks, fuzzy algorithms and other methods are also constantly applied to the automatic control system. Qiao Fuqiang in Tianjin University of Applied Sciences, Tianjin[1] proposed a DSP based on the powerful digital signal processing capability, based on the traditional PID algorithm of automatic control. Since the author added the filter function to the algorithm, the control precision is somewhat improved compared with the traditional method. Krzysztof S. Kula[2] at Gdynia Maritime University established a complete model of ship heading-keeping control, And introduce the neural network method to get the model parameters in order to deal with the ship under different wind and flow conditions; Xingxing Huo of Dalian Maritime University and Chang Jing et al[3][4] from Huanghe Institute of Science and Technology in order to improve the traditional PID algorithm parameter adjustment problems, in their paper were proposed based on BP neural network and PID control algorithm for the automatic control of the rudder. In this method, the PID control parameters are calculated in real time by neural network based on the wind, flow and wave of the current water area of the ship, and the environment adaptability of the autopilot is improved. In order to overcome the defects in the application of the BP neural network, Wang Renqiang and Yuanyuan Wang[5][6] from Australian Maritime University proposed to use RBF neural network to replace BP neural network to calculate PID parameters. Compared with the traditional BP neural network, this control method has faster training and learning speed and can process parallel data at high speed. In order to further improve the intelligent degree of autopilot, Wang Minghui and Yanxiang $\mathrm{Wu}$ [7][8] Are in their papers proposed a fuzzy neural network based on the ship's automatic rudder control algorithm. This algorithm can make use of human knowledge and experience to represent the parameters of the autopilot algorithm with fuzzy algorithm, and also has the advantages of online learning and automatic adjustment of parameters to further improve the practicality of the autopilot; in addition, the research Personnel also carried out research on the autopilot for the ship motion model, but its practicality has been greatly limited due to the great influence of environmental factors on the movement of ships[9].To sum up, using artificial neural network to achieve the automatic control algorithm has become a trend in this

\footnotetext{
+ + Corresponding author. Tel.:+86-27-84226927.

E-mail address: 55440881@qq.com
} 
area and research hot spots. Currently, the DNN method has been successfully applied to image recognition[10-11], speech recognition, language recognition and other fields, and has achieved good results.

\section{Structure for DBN}

DBN consists of a number of structural unit stacks, as shown in Figure 1, the structural unit is usually $\mathrm{RBM}[12]$.

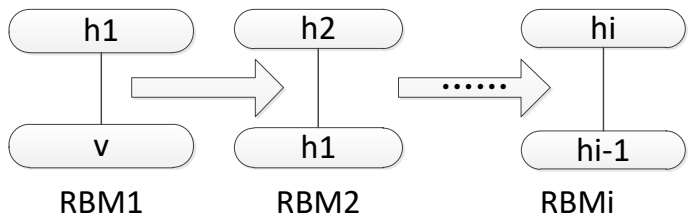

Fig. 1: DBN Structure.

For any RBM in Fig. 1, the structure of the RBM is a two-layer structure model. The bottom layer is a visible layer composed of visible nodes $v=\{v 1, v 2, v 3, \ldots v i\}$. The top layer is composed of hidden nodes $h$ $=\{\mathrm{h} 1, \mathrm{~h} 2, \mathrm{~h} 3, \ldots \mathrm{hj}\}$. The nodes between two layers are fully connected. Each connection has a weight wij, but the nodes in the same layer are not connected to each other. At the same time, the visible layer and hidden layer have their own offset, denoted as a, b. There are two outputs for each neuron, 0 or 1 respectively. The following figure 2 shows the RBM model diagram.

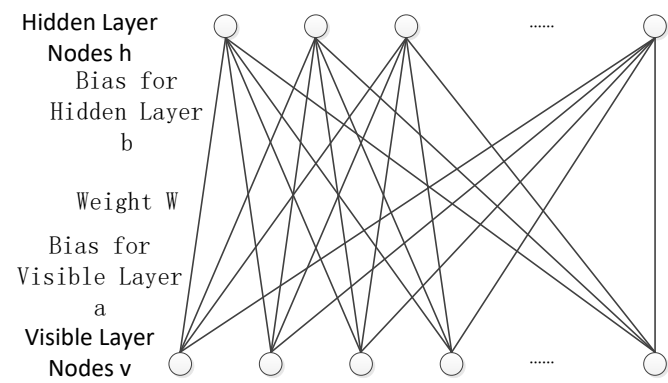

Fig. 2: RBM Model

\subsection{Training Algorithm for DBN}

The DBN model consists of a multi-layered unsupervised RBM and a supervised BP network, combining the advantages of unsupervised learning with supervised learning. DBN training process is divided into pretraining and fine-tuning of two stages [13].

In order to facilitate the later part of the experiment, we present here a RBM model training process based on contrast divergence algorithm. The detailed theoretical basis and derivation of model training can be found in reference[13].

1. Given the training cycle $J$, the learning rate $\eta$, the contrast divergence algorithm in the parameters $\mathrm{K}$ $(\mathrm{K}=1)$ and the number of samples ns.

2. Initialize weight vector $\mathrm{W}$ and offsets $\mathrm{a}, \mathrm{b}$, initialize $\Delta \mathrm{W}=\Delta \mathrm{a}=\Delta \mathrm{b}$.

3. Using the algorithm of contrast divergence algorithm, update $\Delta \mathrm{W}, \Delta \mathrm{a}, \Delta \mathrm{b}$ by circulating samples.

a. Calculate the conditional probability that the k-th neuron in hidden layer is 1 using the following formula (1)

$$
p\left(h_{k}=1 \mid v\right)=\operatorname{sigmoid}\left(b_{k}+\sum_{i=1}^{n} v_{i} * w_{i k}\right)
$$

The result of (1) is compared with the random number $\mathrm{r}$ between $[0,1], \mathrm{hk}=1$ if $\mathrm{r}<\mathrm{P}(\mathrm{hk}=1 \mid \mathrm{v})$, and 0 otherwise. Finally get the updated hidden layer h0.

b. Calculate the conditional probability that the k-th neuron of the visible layer is 1 using the following equation (2), ie

$$
p\left(v_{k}=1 \mid h\right)=\operatorname{sigmoid}\left(a_{k}+\sum_{i=l}^{n} h_{i} * w_{k i}\right)
$$


The result of (2) is compared with the random number $\mathrm{r}$ between $[0,1]$. If $\mathrm{r}<\mathrm{P}(\mathrm{vk}=1 \mid \mathrm{h})$, then $\mathrm{vk}=1$, otherwise 0 . Finally get the updated visual layer v1.

c. Update $\Delta \mathrm{W}, \Delta \mathrm{a}, \Delta \mathrm{b}$ using equations (3) - (5).

$$
\begin{gathered}
\Delta w_{i j}=\Delta w_{i j}+\left[p\left(h_{j}=1 \mid v^{0}\right) v_{i}^{0}-p\left(h_{j} \mid v^{l}\right) v_{i}^{l}\right] \\
\Delta a_{i}=\Delta a_{i}+\left(v_{i}^{0}-v_{i}^{l}\right) \\
\Delta b_{j}=\Delta b_{j}+\left[p\left(h_{j}=1 \mid v^{0}\right)-p\left(h_{j} \mid v^{l}\right)\right]
\end{gathered}
$$

d. Using the algorithm a, b, c three steps to complete the RBM model layer by layer training.

4. Use equations $(6) \sim(8)$ to update the network parameters $\mathrm{W}, \mathrm{a}, \mathrm{b}$.

$$
\begin{gathered}
W=\eta\left(\frac{1}{n_{s}} \Delta W\right) \\
a=\eta\left(\frac{1}{n_{s}} \Delta a\right) \\
b=\eta\left(\frac{1}{n_{s}} \Delta b\right)
\end{gathered}
$$

5. Enter algorithm step 2 until the training period is met, then the algorithm stops.

The parameters trained in the above method need to be reversely tuned to achieve the overall optimal effect of DBN. In the case of reverse fine-tuning, DBN uses BP network to supervise the top-level BP network and classifies the features learned by RBM network. The error between DBN actual output and expected output is reversely transmitted to all RBM networks, Fine-tune the parameters between RBM network layer, get the best DBN.

\section{Key Technology for Model Building}

\subsection{Input Parameters}

According to the design principle of sailing simulator, the influencing factors with greater weight are chosen as the training input of neural network, such as wind direction, wind force, flow direction, flow rate and so on. Because the DBN model can only identify the two kinds of data of 0 and 1 , all the factors that affect the movement of the ship are converted into the binary numerical form.

\subsection{Output Parameters}

The function and function of DBN is to realize the change of rudder angle so as to make the ship sailing on the target route as stable as possible. Therefore, there is only one output parameter of the neural network, which is the target rudder angle. For ordinary merchant ships, the value of the rudder angle is generally between $-35^{\circ}$ and $+35^{\circ}$. Therefore, the output value of the network is located between 0 and 71 and expressed in 7-bit binary.

\section{Model Analysis and Verification}

\subsection{Model Structure}

Figure 3 below shows the most widely used 5-layer DBN model. 


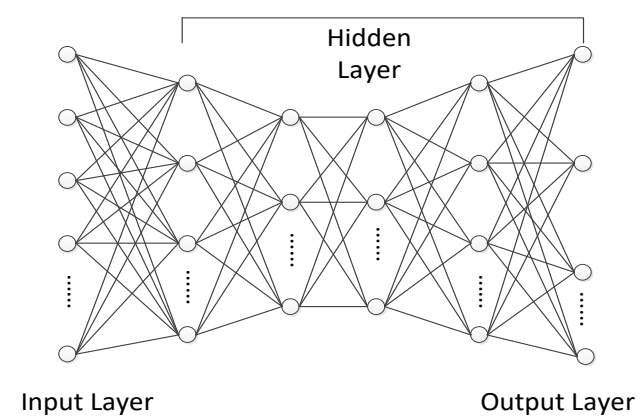

Fig. 3: DBN Model with 5 Layers

Based on the DBN model as shown in Figure 3 above, DeepLearnToolbox [14] was introduced as a development tool to fine-tune the number of hidden layer neurons to verify the effect of different numbers of neurons on DBN model.

\subsection{Data analysis and Comparison}

The number of the ship in the simulation experiment is No. 3, the length is 179.7 meters, the breadth is 27.6 meters and the maximum draft is 9.5 meters. The ship type is a container ship. Shiping scene is anchored outside the mouth of the Yangtze River into Hong Kong, the data recording time of 500s, the initial value of 0 to the bow, does not involve collision avoidance and super-ship. The manual steering and the autopilot have been separately recorded, as shown in Figure 4 and Figure 5 below.
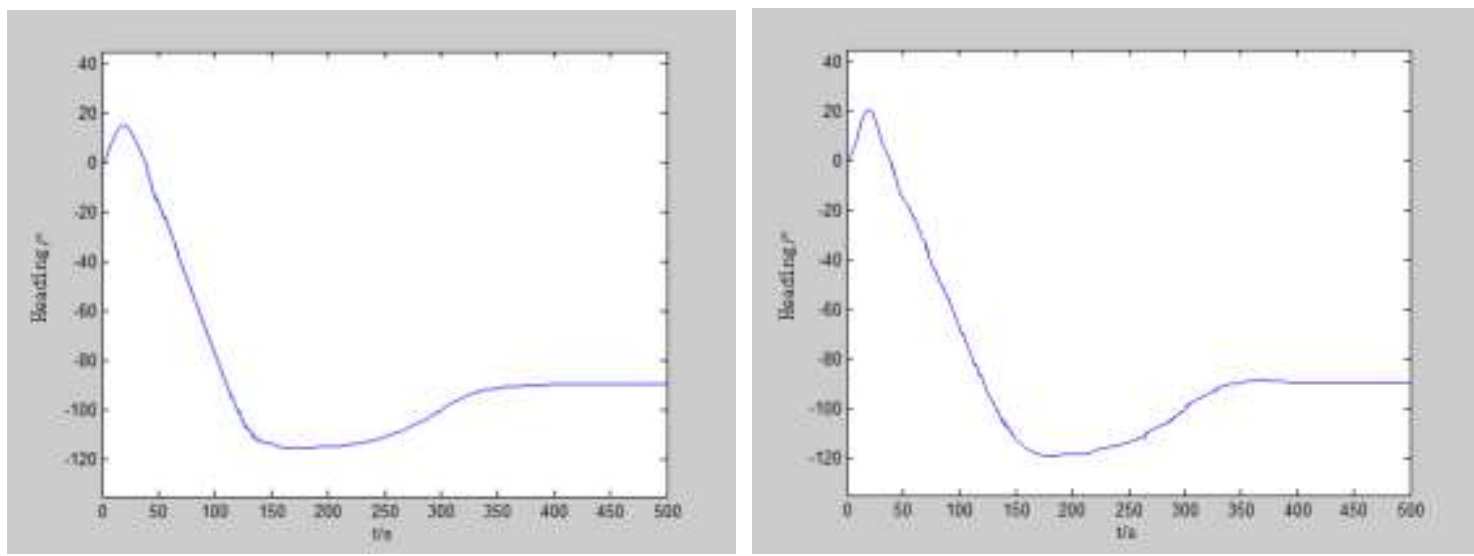

Fig. 4: Curve for Ship Heading(Left for Man/Right for Machine).
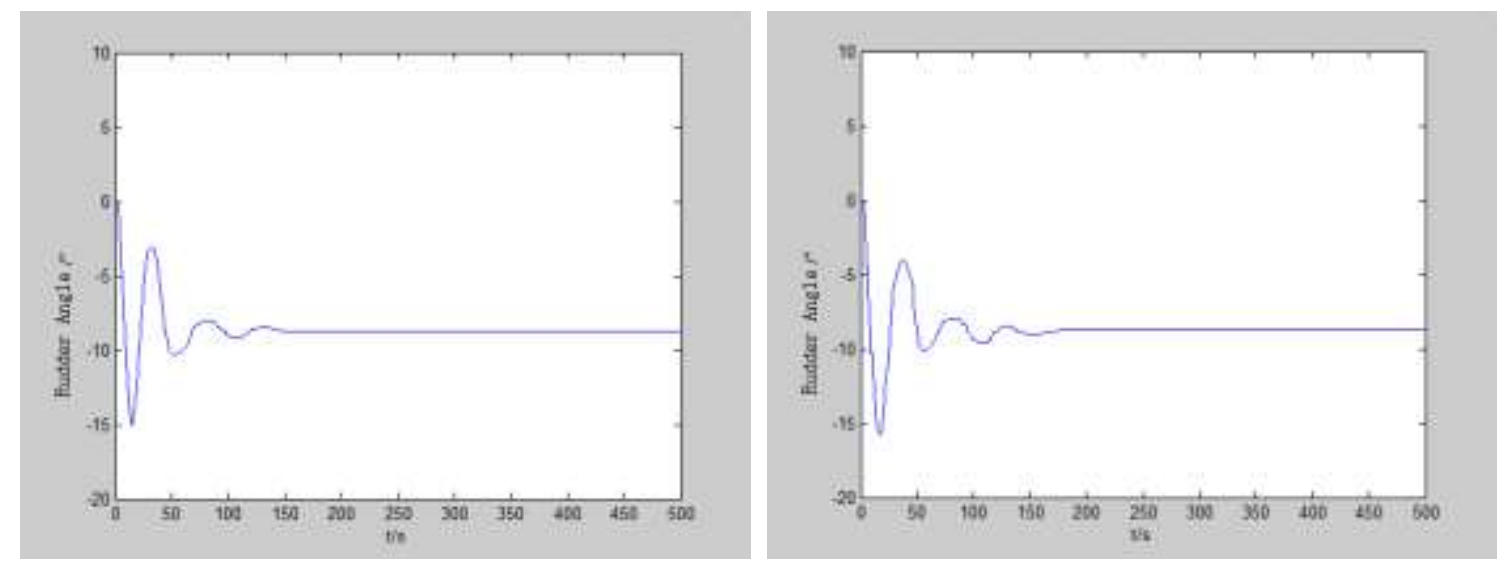

Fig. 5: Curve for Rudder(Left for Man/Right for Machine).

From Figure 4 and Figure 5 we can see that the machine maneuvering and artificial maneuvering in the accuracy is very close. In order to further analyze the error, we choose to make an error record once per second, denoted as ei, unit meter, and use the following equation (9) (10) to get the error index. 


$$
\begin{gathered}
E=\frac{\sum_{i=0}^{n} e_{i}}{n} \\
D=\frac{\sum_{i=0}^{n}\left(e_{i}-E\right)^{2}}{n}
\end{gathered}
$$

where $\mathrm{E}$ is the mean of the error and $\mathrm{D}$ is the variance of the error. After calculation, we can see that the error of manual handling is 22.5 and the variance is 4.6 . The error of machine handling is 23.7 and the variance is 5.3. The above data also shows that the autopilot based on DBN model has higher accuracy.

On the contrary, the existing related control algorithms, most of the authors of the article only put forward the relevant theory, and did not verify or simulate the relevant theory [3][4][5-8].

\section{Conclusion}

In this paper, an automatic steering control system based on DBN is proposed in this paper. The hidden layer is defined as five layers. At the same time, the test training data recorded by Shanghai Maritime University's Advanced Crew Training System is used as a training sample. Divergence algorithm, the training of each layer RBM composed of DBN model in order to obtain the initial value of DBN; and then extracted the training samples in the actual operation accuracy of the data as labeled data, the use of BP algorithm to achieve the DBN Fine-tuning. In order to improve the development efficiency, DeepLearnToolbox tool is introduced in this paper to realize the rapid training of DBN model.

The simulation results show that the difference between the control accuracy and the artificial steering accuracy of this algorithm is very small, with an average error of only $5.2 \%$, which has already possessed the basic conditions of real ship test.

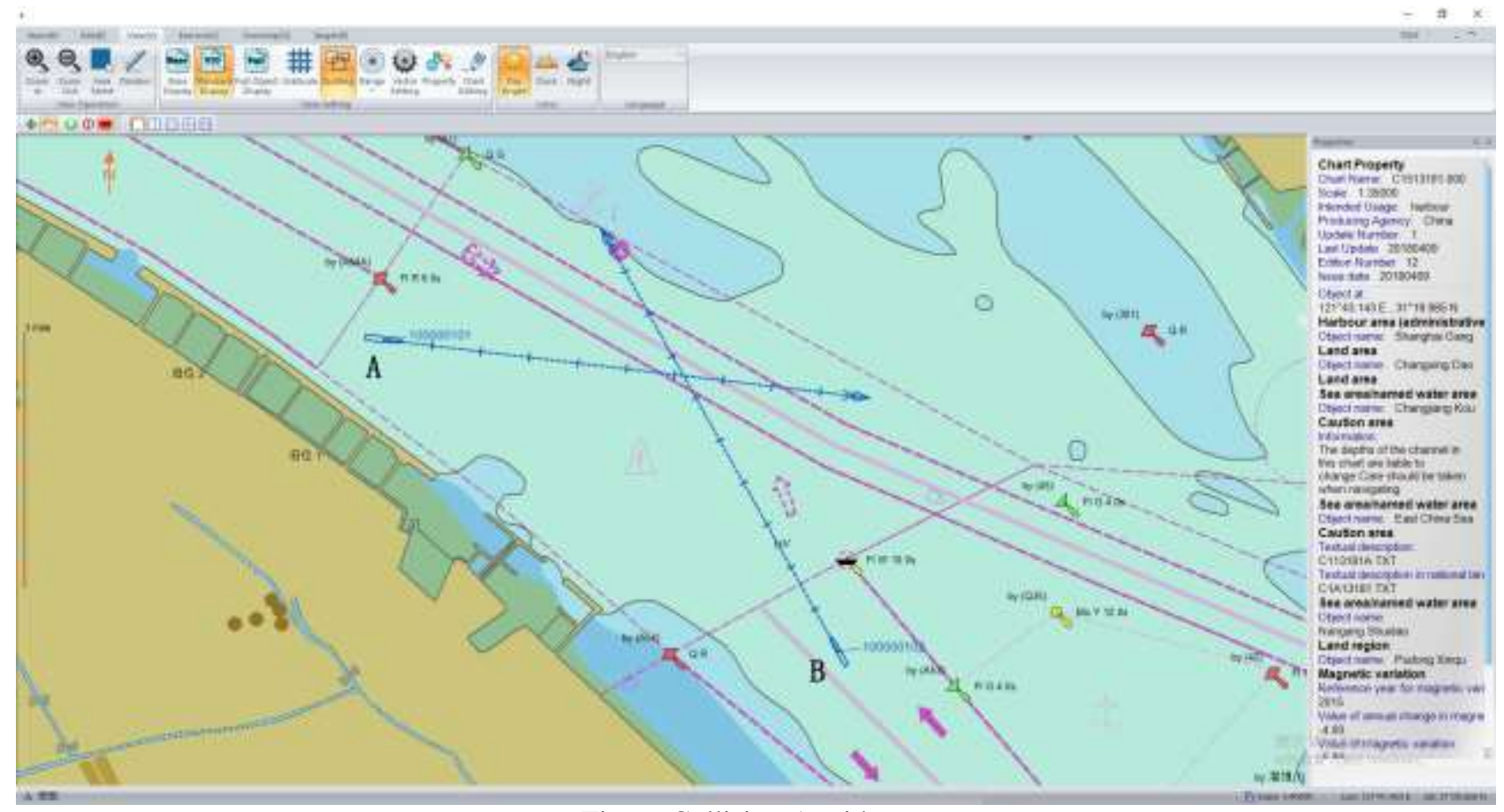

Fig. 6: Collision Avoidance.

\section{Acknowledgement}

We gratefully acknowledge the support funding by guiding item of research project of Hubei provincial department of education (B2018254), as well as help from Shanghai Maritime University.

\section{References:}

[1] Qiao Fuqiang. Research on PID automatic rudder monitoring system based on DSP Technology[J]. Ship Science and Technology, 2017, 39(7A):127-129 
[2] Krzysztof S. Kula. Model-based controller for ship track-keeping using neural network[C]// 2nd International Conference on Cybernetics. Poland: IEEE Press,2015:178-183

[3] HUO Xing-xing, HU Jiang-qiang, YU Ting. Fuzzy PID autopilot for ship steering based on stratification and sub fuzzy controller[J]. Journal of Dalian Maritime University,2015,39(4):71-74

[4] Chang Jing. Application and research of the fuzzy PID autopilot based on BP neural network[J]. Ship Science and Technology, 2017,39(3A):105-107

[5] Wang Renqiang, Zhao Yuelin, Sun Jianming. Application of Optimized RBF Neural Network in Ship's Autopilot Design[C]// 2016 IEEE Advanced Information Management, Communicates, Electronic and Automation Control Conference. Xi’an:IEEE Press, 2016:1642-1646

[6] Yuanyuan Wang, Hung Duc Nguyen, Shuhong Chai and Faisal Khan. Radial Basis Function Neural Network Based Rudder Roll Stabilization for Ship Sailing in Waves[C]// 2015 5th Australian Control Conference. Gold Coast ,Australia:IEEE Press,2015:158-163

[7] Wang Minghui, Yu Yongquan, Zeng Bi. Study of Intelligent Control System for Ship Autopilot Design[C]// 2009 Eigth IEEE/ACIS International Conference on Computer and Information Science, Shanghai, China: IEEE Press,2009:701-705

[8] Yanxiang Wu,Minjie Xue,Pengjie Wu. Study on fuzzy neural network-based ship autopilot[C]//International Conference on Neural Computation, Yantai, China: IEEE Press,2010: 1393-1396

[9] Kapitanyuk, Yuri A. Optimal controllers for rudder roll damping with an autopilot in the loop[J]. IFACPapersOnLine, 2016,49(3):562-567

[10] HINTON G E, OSINDERO S, TEH Y W. A fast learning algorithm for deep belief nets [J] . Neural Computation, 2006, 18(7):1527-1554.

[11] BENGIO Y. Learning deep architectures for AI [ J . Foundations and Trends in Machine Learning, 2009, 2(1):1-127.

[12] Liu Jianwei, Liu Yuan, Luo Xionglin. Research and development on deep learning[J]. Application Research of Computers,2014,34(7):1921-1930,1942

[13] LAROCHELLE H, BENGIO Y, LOURADOUR J, et al. Exploring strategies for training deep neural networks[J] . Journal of Machine Learning Research, 2009, 10(12):1-40.

[14] Rasmus Berg Palm. DeepLearnToolbox-Master[EB/OL].https://github.com/rasmusbergpalm/DeepLearnToolbox, 2012. 